\title{
RELATIONSHIP BETWEEN SHEAR BOND STRENGTH AND FLUORIDE CONTENT OF FLUORIDATED TEETH AS STATED BY SCANNING ELECTRON MICROSCOPY AND X-RAY FLUORESCENCE ANALYSIS
}

\author{
Samy M El-Safty* and Tasneem A Almohamady ${ }^{* *}$
}

\begin{abstract}
Objectives: To study the effect of fluoride treatment on shear bond strength (SBS) of orthodontic brackets and to investigate the relationship between SBS and fluoride content of fluoridated teeth by means of scanning electron microscopy (SEM) and X-ray fluorescence (XRF) analysis.

Materials and methods: A total of one hundred sound maxillary premolars were thoroughly cleaned and randomly assigned to 5 groups $(n=20)$ : control group without fluoride treatment and four groups fluoridated with Profluorid ${ }^{\circledR}$ Varnish $5 \% \mathrm{NaF}$ (VOCO GmbH. Cuxhaven, Germany) and stored in artificial saliva for varied periods: 1 day, 3 days, 5 days and 7 days. After completing the predetermined storage time, specimens of each group were subdivided into two subgroups $(n=10)$; ten specimens were used for SBS testing and the other ten specimens were submitted to XRF analysis to study their fluoride content. An etchant (phosphoric acid 37\%) and a resin adhesive system Transbond XT (TXT) (3M ESPE, St. Paul, Minn) were used to bond the ceramic brackets (Perfect Clear, Orthodontic Sapphire Ceramic Brackets, HUBIT Co., Ltd. South Korea) to the teeth surfaces. Debonding was carried out in a universal testing machine (Lloyd, Type 500, Lloyd Instrument, England) at a cross-head speed of $1 \mathrm{~mm} / \mathrm{min}$. After debonding, a stereomicroscope (Olympus SZ 60, Japan) was used to determine the type of fracture of each specimen and scores of adhesive remnant index (ARI) were given. SEM investigation was conducted on representative specimens of debonded tooth surfaces of all groups in a scanning electron microscope (Jeol, JSM5200LV scanning microscope, Japan). XRF analysis was carried out using AXIOS, WD-XRF Sequential Spectrometer (Malvern Panalytical, 2005, United Kingdom). Crowns of specimens of each subgroup were cut, ground and pressed by the aid of binding wax to form disc-shaped specimens ( $35 \mathrm{~mm}$ in diameter $\times 3 \mathrm{~mm}$ in thickness) that were submitted to the XRF analysis. Oneway ANOVA, Tukey test and chi-square test were used to analyze the data. Significance level was established at $(\mathrm{p} \leq 0.05)$.
\end{abstract}

Lecturer, Department of Dental Biomaterials, Faculty of Dentistry, Tanta University, Egypt.

** Orthodontist, El-Santa Central Hospital, El-Santa, Gharbia, Egypt. 
Results: statistically significant differences were found between studied groups in both SBS values and ARI scores. SEM examination showed that fluoride layer acted as a barrier hindering proper etching of tooth surface that resulted in poorer bonding compared to control group. Longer storage, however, caused fluoride layer to be washed away giving better bonding. $\mathrm{XRF}$ analysis revealed greater fluoride content of groups with shorter storage time compared to those with longer storage. Linear regression analysis revealed a strong positive correlation $\left(\mathrm{R}^{2}=0.936\right)$ between SBS and storage time and a strong negative correlation $\left(\mathrm{R}^{2}=0.959\right)$ between SBS and fluoride content of fluoridated groups.

Conclusions: As confirmed by SEM and XRF analysis, fluoride reduced SBS of orthodontic brackets in the first few days of fluoride treatment. As fluoride dissolved in artificial saliva after a period of time, SBS recovered to normal values.

Clinical significance: For patients who are in need to orthodontic treatment, if fluoride treatment is mandatory, bonding of orthodontic brackets should be postponed seven days after treatment to get adequate bonding to the tooth structure.

Keywords: Shear Bond Strength; Topical Fluoride; Fluoride Content; X-ray Fluorescence; Scanning Electron Microscopy.

\section{INTRODUCTION}

Achievement of successful bonding between the tooth structure and restorative dental materials is of great importance in dental practice ${ }^{[1,2]}$. Such desirable bonding should be strong enough to withstand polymerization contraction stresses of some restorative materials, such as resin-composite, that may weaken or cause failure of the bond, provide a strengthening effect on remaining tooth structure and ensure perfect sealing against ingress of oral fluids and bacteria, thus preventing demineralization and caries of tooth structures ${ }^{[3]}$. The quality of orthodontic treatment is greatly influenced by proper bonding of orthodontic brackets to enamel surface and long-term retention of the accessories. This is because failure of bracket bonding can disrupt, increase time and costs of treatment and hinder the correct finalization of the case. In addition, repeated bracket rebonding can cause damage to enamel structure ${ }^{[4,5]}$.
In orthodontic treatment, however, some dental and periodontal problems may arise. Unaesthetic decalcifications and white spot lesions under and around orthodontic brackets and bands may be formed. It was reported that, in the absence of proper oral hygiene and plaque control, clinically visible white spot decalcifications can develop around fixed orthodontic appliances as early as four weeks after placement. In addition, some studies reported that incidence of white spot lesions during orthodontic treatment was as high as one third of patients undergoing orthodontic treatment ${ }^{[6]}$.

Therefore, some researchers and clinicians recommended fluoride application for preventing cariogenic effects. To fluoridate a tooth surface, one of three approaches can be utilized; i) pretreatment with topical fluoride before acid etching, ii) application of fluoride after etching the tooth surface but before the adhesive application, and iii) incorporating fluoride into the etching agent, the adhesive or the resin-composite ${ }^{[7]}$. An interpretation of the effect of fluoride on tooth surface is based on the formation of fluoroapatite that has been shown to be more resistant to acidic dissolution than nonfluoridated hydroxyapatite ${ }^{[8]}$. Another possibility is that when a fluoride is applied to the enamel surface, a precipitation of globules of calcium fluoride $\left(\mathrm{CaF}_{2}\right)$ known as phosphate-contaminated calcium fluoride takes place. This calcium fluoride layer is a key factor in caries prevention ${ }^{[9]}$. When precipitated onto the enamel surface, $\mathrm{CaF}_{2}$ globules can penetrates into the inter-prismatic spaces, occupied by water and proteins, reducing the enamel permeability and, therefore, the movement of fluids ${ }^{[10]}$. Unfavorably, reduction of enamel permeability may result in limited penetration by the adhesive and shorter resin tags compared to that of untreated enamel surfaces ${ }^{[11]}$.

Several studies have been conducted to investigate the effect of fluoride application on bond strength of orthodontic brackets. Some studies reported significantly increased bond strength ${ }^{[12]}$, 
others found that fluoride caused an adverse effect ${ }^{[13,14]}$ and a third category ${ }^{[15,16]}$ reported no effect. Nevertheless, to the best of our knowledge, no studies have been found investigating the correlation between the bond strength value of the fluoridated teeth and their fluoride content.

Analysis of elements, qualitatively and quantitatively, in environmental, biological, medical and dental specimens can be performed by a variety of methods ${ }^{[17]}$. X-ray fluorescence (XRF) analysis is one of these methods that can be applied to determine the chemical composition of all types of materials that can be in solid, liquid, powder or other form. It can be also useful in determination of thickness and composition of layers and coatings. It utilizes characteristic X-rays known as "fluorescence $\mathrm{X}$-rays" emitted under high-energy X-ray irradiation. This method is characterized by being fast, accurate and non-destructive and usually requires a minimum of sample preparation. In research, XRF is a very useful analytical technique by which a variety of materials can be analyzed. These include metals, cements, polymers, oil, plastics, food industries along with geology and environmental analysis of water and waste materials ${ }^{[18]}$.

By the aid of XRF analysis, Baranowska et al. ${ }^{[19]}$, found a positive correlation between the level of pollution in the environment and $\mathrm{Zn}, \mathrm{S}$ and $\mathrm{Pb}$ concentrations in teeth derived from inhabitants from the most polluted and less polluted areas in Poland. In addition, they reported significantly increased levels of $\mathrm{Zn}$ and $\mathrm{Pb}$ in teeth from smokers compared to those of non-smokers. In this study, we tried to apply the XRF analysis to determine the fluoride concentrations in teeth subjected to topical fluoride application for different periods. Therefore, the main objective of this study was to study the correlation between the shear bond strength (SBS) of teeth subjected to fluoride application and their fluoride content by means of SEM and XRF analysis. The null hypotheses of our study were: i) there will be no significant difference between SBS of fluoridated and non-fluoridated teeth and ii) there will be no correlation between SBS of fluoridated teeth and their fluoride content.

\section{MATERIALS AND METHODS}

\section{Teeth collection and preparation:}

The study design was approved by Research Ethics Committee, Faculty of Dentistry, Tanta University. The purpose of the study was explained to the patients and informed consents were obtained to use their extracted teeth in the research according to the guidelines on human research adopted by Research Ethics Committee, Faculty of Dentistry, Tanta University.

A total of one hundred maxillary premolars were collected from Orthodontics and Oral Surgery departments. The teeth were thoroughly examined so that any restored premolars or those with enamel delamination, defects or cracks were excluded. Premolars with noticeable deformity in shape or size or those subjected to any chemical treatment that can affect enamel such as alcohol, ethanol or hydrogen peroxide were excluded as well. After extraction, teeth were cleaned of organic remnants, washed, and placed in $0.1 \%$ thymol solution (Thymol Mylan, Seiyaku, Japan) to prevent bacterial growth and dehydration. Before use, their buccal surfaces were cleaned with a rubber polishing cup fitted to a dental contra-angle hand-piece with aqueous refrigeration. The teeth were used within six months of extraction as recommended by ISO standards ${ }^{[20]}$.

\section{Grouping}

According to the topical fluoride application and storage time (after fluoride application and before bonding), the collected teeth were divided into five groups, one control without fluoride treatment and four fluoridated groups, twenty specimens per each $(n=20)$. In the control group, the teeth were stored in artificial saliva for $24 \mathrm{~h}$ at $37^{\circ} \mathrm{C}$. In treated groups, a fluoride varnish - Profluorid ${ }^{\circledR}$ Varnish 5\% $\mathrm{NaF}$ (VOCO GmbH. Cuxhaven, Germany) - was 
applied to the buccal surfaces of teeth and stored in artificial saliva for one, three, five and seven days before bonding to orthodontic brackets. The fluoride varnish was applied as a thin film to the enamel of buccal surfaces with a disposable applicator and left to penetrate the surface for $20 \mathrm{~s}$, and then dried with oil-free compressed air before storage. Fluoridated teeth were stored in artificial saliva for a pre-determined time according to the study design and kept at a temperature of $37^{\circ} \mathrm{C}$, in an incubator (JP Selecta S.A., Barcelona, Spain) to ensure proper setting of fluoride varnish. The artificial saliva used as a storage medium was composed of $1 \%$ carmellose sodium, $13 \%$ sorbitol, $0.084 \%$ sodium chloride, $0.12 \%$ potassium chloride, $0.005 \%$ magnesium chloride hexahydrate, $0.017 \%$ dibasic potassium phosphate, $0.015 \%$ anhydrous calcium chloride and $0.1 \%$ Nigapin ${ }^{\circledR}$ sodium. The saliva $\mathrm{pH}$ was adjusted and maintained at $6.57^{[7]}$.

\section{Bonding Procedures}

After completing the storage time pre-determined for each group, specimens of each group were divided into two halves: ten specimens were bonded to the brackets and submitted to SBS testing and the other ten specimens were used for the XRF analysis. For bond strength testing, the buccal surfaces of teeth were cleaned with distilled water spray and dried using oil-free compressed air. The buccal enamel surfaces of all teeth (control and fluoridated) were etched with $37 \%$ phosphoric acid for $30 \mathrm{~s}$, rinsed for $15 \mathrm{~s}$ and dried for $20 \mathrm{~s}$. Specimens of all groups were bonded to the ceramic Roth brackets (Perfect Clear, Orthodontic Sapphire Ceramic Brackets, HUBIT Co., Ltd. South Korea) with beads base design and bracket base area of 10.5 $\mathrm{mm}^{2}$ using a conventional resin adhesive Transbond XT (TXT) (3M ESPE, St. Paul, Minn). Its chemical composition is made up of bisphenol A diglycidyl ether dimethacrylate, bisphenol A bis (2-hydroxyethyl ether) dimethacrylate, silane-treated silica and silane-treated quartz.
The TXT primer was applied to the bonding area with a disposable applicator rubbing the surface for $20 \mathrm{~s}$. The solvent was eliminated by drying the surface with oil-free compressed air for $5 \mathrm{~s}$ and then photopolymerized with an LED light curing unit (Woodpeker, China) with irradiance of $1200 \mathrm{~mW} /$ $\mathrm{cm}^{2}$ for $10 \mathrm{~s}$. Following this, the TXT adhesive was applied to the bracket base. Each bracket was positioned on the buccal surface at the height of contour mesio-distally, in the middle third occlusogingivally and parallel to the long axis of the tooth using bracket placement tweezers and subjected to $300 \mathrm{gm}$ compressive force using a correx force gauge (Hensgrand, China) for $10 \mathrm{~s}$ to ensure a uniform adhesive thickness between the bracket and tooth surface ${ }^{[21]}$. Excess adhesive material was removed using a sharp scaler and then the adhesive was light-cured for $20 \mathrm{~s}$ from the incisal edge and $20 \mathrm{~s}$ from the gingival edge of the attached bracket. The teeth were then stored in distilled water at $37^{\circ} \mathrm{C}$ for $24 \mathrm{~h}$ before bond strength testing ${ }^{[20]}$.

\section{Shear bond strength testing}

To facilitate testing, specimens of each group (control and fluoridated) were embedded in an autopolymerizing polymethyl methacrylate (PMMA; Esschem Co., PA, USA). PMMA was mixed according to the instructions of the manufacturer and poured into a cylindrical mold measuring $4 \mathrm{~cm}$ in diameter and $3 \mathrm{~cm}$ in depth. The embedding process of teeth was done carefully so that the crown of each tooth was clearly exposed for easy testing.

Each specimen was mounted on the lower fixed compartment of a computer-controlled universal testing machine (Lloyd, Type 500, Lloyd Instrument, England). At a cross-head speed of $1 \mathrm{~mm} / \mathrm{min}$, each specimen was subjected to a compressive load via a mono-beveled chisel attached to the upper movable compartment of the testing machine. Care was taken so that the chisel tip was positioned to touch only the base of the brackets and be parallel to the long axis of each mounted tooth. The load required to dislodge each bracket was recorded in 
newtons $(\mathrm{N})$ and the tested SBS was calculated in megapascals $(\mathrm{MPa})$ by dividing the load in Newton (N) by the surface area of the bracket base $(10.5$ $\mathrm{mm}^{2}$ ) as given by the manufacturer. Debonding was manifested by bracket displacement and confirmed by sudden drop along the load-deflection curve recorded by the computer software (Nexygen-MT; Lloyd Instruments Ltd).

\section{Determination of fracture type and adhesive remnant index (ARI)}

At a magnification of $20 \mathrm{x}$, a stereomicroscope (Olympus SZ 60, Japan) was used to determine the type of fracture that was one of the following categories: i) cohesive failure in enamel; ii) cohesive failure in adhesive; iii) adhesive failure at tooth/ adhesive interface; iv) adhesive failure at bracket/ adhesive interface and v) mixed failure (partial cohesive and partial adhesive). The amount of adhesive remaining was assessed with ARI scores which were developed by Artun and Bergland [22] and modified by Lalani et al. ${ }^{[23]}$ as follows:

Score 0: indicates no adhesive remained on the tooth.

Score 1: less than $50 \%$ of the adhesive remained on the tooth.

Score 2: more than $50 \%$ of the adhesive remained on the tooth.

Score 3: all adhesive remained on the tooth with distinct impression of the bracket mesh on the tooth surface.

\section{Scanning electron microscopy (SEM)}

Scanning electron microscopy (SEM) was conducted on representative specimens of debonded tooth surfaces of all groups to observe surface changes and effect of fluoride application. The buccal surface of each tooth was cleaned with distilled water spray, dried, and left at room temperature to dry. The crown of each premolar was sectioned from the roots using a low speed double sided- diamond disc under continuous water spray irrigation. Then each tooth was cut on a mesiodistal line starting from the occlusal side until reaching the cervical line. The buccal surface was retained for SEM examination. All specimens were mounted on stubs and prepared for SEM by sputtering with gold, then examined in a scanning electron microscope (Jeol, JSM-5200LV scanning microscope, Japan) operated at $15 \mathrm{KJ}$. At a magnification of $500 \mathrm{x}$, the buccal surfaces of specimens were examined - at the orthodontic bonding area- to obtain representive photomicrographs.

\section{X-ray fluorescence (XRF) analysis}

$\mathrm{X}$-ray fluorescence spectrometry (XRFS) is a method of elemental analysis that assesses the presence and concentration of various elements. The basic concept of all spectrometers is a source, a sample and a detection system. The source irradiates a sample and a detector measures the radiation coming from the sample. This investigation was conducted at the XRF lab of the National Research Center (NRC), Cairo, Egypt and procedures were carried out according to ASTM E1621-2013 ${ }^{[24]}$. The crowns of 10 specimens from each group were crushed then ground in a grinding machine (FRITSCH Mortar Grinder Pulverisette 2, FRITSCH, Germany) to get a fine powder. Disc-like samples ( $35 \mathrm{~mm}$ in diameter $\times 3 \mathrm{~mm}$ in thickness) were prepared from this powder by manual mixing of $6 \mathrm{gm}$ of the powder of each group with $1.5 \mathrm{gm}$ of a wax binding material for one minute. The powder of each sample was then pelletized in an aluminum cup and pressed in an automatic pressing machine (Siemens Simatic C7-621, Herzog, Germany) under $130 \mathrm{kN}$. The pelletized samples were then used in qualitative and quantitative analysis of the required elements. In the current study, determination of fluoride ions in each specimen was our main objective.

XRF spectrometer systems are divided into two main types: energy dispersive systems (ED-XRF) and wavelength dispersive systems (WD-XRF). 
In the current study, the XRF analysis was conducted using AXIOS, WD-XRF Sequential Spectrometer (Malvern Panalytical, 2005, United Kingdom). It is an instrument with a single goniometer basedmeasuring channel covering the complete measured range. The instrument is a microprocessor controlled by an external computer with an analytical software package (Super Q 4). Concentration of various elements could be estimated by the measurement of secondary $\mathrm{x}$ - radiation from the specimens that were excited by an $x$-ray source.

\section{Statistical analysis}

Data were collected and tabulated and statistically analyzed by an IBM compatible personal computer with SPSS version 20 (SPSS Inc. Released 2011, Armnok, NY: IBM Corp). Data of SBS were analyzed using a one-way analysis of variance (ANOVA) with the significance level established at $(\mathrm{p} \leq 0.05)$. The post-hoc Tukey test was used to determine differences between groups. The chi-square test was used to evaluate statistically significant differences in the frequencies of ARI scores between groups. Linear regression analysis was conducted to investigate any relationship between the SBS of fluoridated teeth and their fluoride content as revealed by XRF analysis.

\section{RESULTS}

\section{Shear bond strength (SBS)}

Means and standard deviations of shear bond strength (SBS) of all studied groups are listed in Table 1 and shown in Figure 1. Results of multiple comparisons are shown in Table 1 by superscript letters as well, $(\mathrm{p} \leq 0.05)$.

The control (non-fluoridated) group showed a highly significantly greater SBS than those recorded by Day1, Day3 and Day5 groups $(p=0.000)$ but had no significant difference with Day7 group ( $\mathrm{p}$ $=0.773)$. In fluoridated groups, results revealed a systematic increase in SBS with increasing the storage time, where Day7 group recorded greater SBS strength than Day5, Day3 and Day1 groups. Day5 group exhibited greater SBS than those of Day3 and Day1 groups and Day3 group showed greater SBS than that of Day1 group. Upon storing the fluoridated teeth for 7 days, SBS was comparable to that of the control group. A linear regression analysis between SBS and days of storage of fluoridated groups demonstrated a strong positive correlation $\left(\mathrm{R}^{2}=0.936\right)$ as shown in Figure 2.

TABLE (1) Mean and standard deviation of shear bond strength (MPa) of control and fluoridated groups. Each value represents the mean of ten measurements. Different superscript letters mean statistically significant $(\mathrm{p} \leq 0.05)$.

\begin{tabular}{|c|c|c|c|}
\hline \multicolumn{4}{|c|}{ Shear Bond Strength (MPa) } \\
\hline Groups & Mean (SD) & F & p-value \\
\hline Control & $17.87(0.99)^{\mathrm{a}}$ & & \\
\hline Day 1 & $6.61(1.23)^{\mathrm{b}}$ & & \multirow{2}{*}{252.394} \\
\cline { 1 - 2 } Day 3 & $7.14(1.19)^{\mathrm{b}}$ & & \\
\hline Day 5 & $12.17(1.11)^{\mathrm{c}}$ & & \\
\hline Day 7 & $17.28(1.09)^{\mathrm{a}}$ & & \\
\hline
\end{tabular}

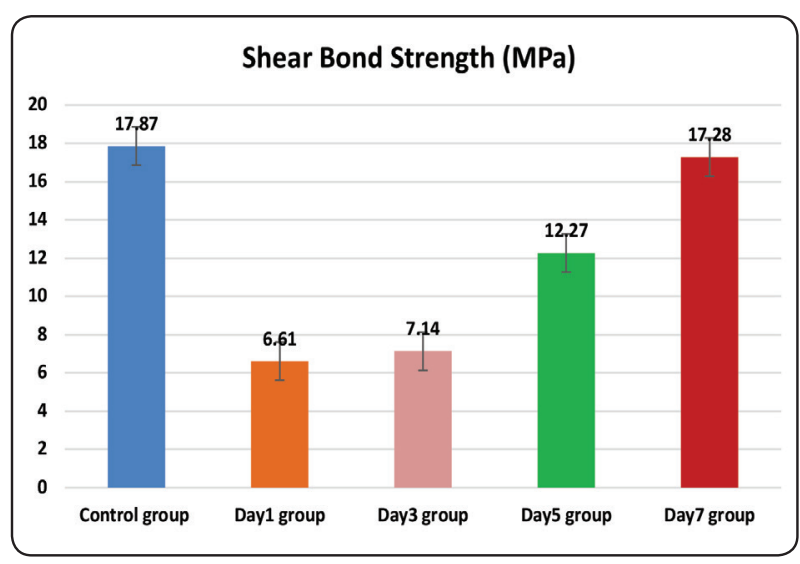

Fig. (1) Bond strength (MPa) of control and fluoridated groups. Each value represents the mean of ten measurements. 


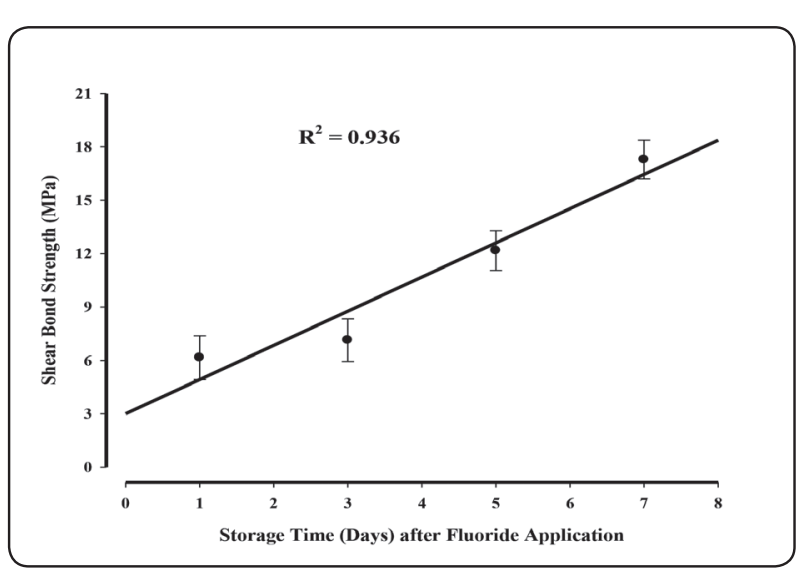

Fig. (2) Within the storage period selected, linear regression analysis showed a strong positive correlation $\left(\mathrm{R}^{2}\right.$ $=0.936)$ between shear bond strength $(\mathrm{MPa})$ of fluoridated groups and storage time (in days) after fluoride application.

\section{Adhesive remnant index (ARI)}

Results of ARI are presented in Table 2 and shown in Figure 3. The chi-square analysis revealed significant differences between ARI scores of studied groups. The control group showed significant differences with all fluoridated groups except Day7 group. Also, there were no significant differences between Day1 and Day3 groups. The greatest ARI frequency for control and Day7 groups was at score 2 (70\% and 60\%, respectively). For Day1 and Day3 groups, the greatest ARI frequency was at score 0 ( $80 \%$ and $70 \%$, respectively). Day5 showed the highest ARI frequency at score $1(70 \%)$.

\section{Scanning electron microscopy (SEM)}

Scanning electron micrographs of all groups are shown in Figure 4. These graphs almost confirmed the findings of SBS testing and ARI analysis. In control group, the etched enamel surface revealed a honeycomb appearance characteristic of good bonding between tooth surface and adhesive because of penetration of resin into the micropores created on the enamel surface. In Day1, Day3 and Day5 groups, the scanning revealed a layer of applied fluoride varnish acting as a barrier between the tooth surface and etching acid. However, groups with longer storage time showed more dissolution of the fluoride layer and increased roughness on the enamel surface compared to those with shorter storage time. In case of Day7 group, the tooth surface was entirely uncovered because of nearly complete dissolution of the fluoride layer and a honeycomb appearance like that in the control group was evident.

\section{X-ray fluorescence (XRF) analysis}

Results of the XRF analysis are presented in Table 3. The main constituents of the examined tooth samples are presented by their weight percentage (wt\%). The fluoride content of the fluoridated groups decreased systematically with increasing the time of storage, where the greatest fluoride content was shown by Day1 group and the lowest was recorded for Day7 group. The control group, however, showed no fluoride ions. For fluoridated groups, linear regression analysis revealed a strong negative correlation $\left(R^{2}=0.959\right)$ between SBS and fluoride content, Figure 5.

TABLE (2) Frequency distribution of the Adhesive Remnant Index (ARI) Scores (\%) for the studied groups.

\begin{tabular}{|c|c|c|c|c|c|}
\hline \multicolumn{6}{|c|}{ Adhesive Remnant Index (ARI) } \\
\hline Groups & Score 0 & Score 1 & Score 2 & $\mathrm{~F}$ & p-value \\
\hline Control & $10 \%(1 / 10)$ & $20 \%(2 / 10)$ & $70 \%(7 / 10)$ & \multirow{5}{*}{33.221} & \multirow{5}{*}{$0.000^{* *}$} \\
\hline Day 1 & $80 \%(8 / 10)$ & $20 \%(2 / 10)$ & - & & \\
\hline Day 3 & $70 \%(7 / 10)$ & $30 \%(3 / 10)$ & - & & \\
\hline Day 5 & $30 \%(3 / 10)$ & $70 \%(7 / 10)$ & - & & \\
\hline Day 7 & $10 \%(1 / 10)$ & $30 \%(3 / 10)$ & $60 \%(6 / 10)$ & & \\
\hline
\end{tabular}


TABLE (3) Main constituents of the studied tooth samples and their weight percentages as stated by the $\mathrm{XRF}$ analysis.

\begin{tabular}{|c|c|c|c|c|c|}
\hline Groups & Control & Day1 & Day3 & Day5 & Day7 \\
\hline Constituents & \multicolumn{5}{|c|}{$(\mathrm{Wt} \%)$} \\
\hline $\mathrm{Ca}$ & 60.51 & 60.73 & 62.42 & 61.14 & 60.97 \\
\hline $\mathrm{P}$ & 21.96 & 22.04 & 22.44 & 21.09 & 21.95 \\
\hline $\mathrm{Si}$ & 0.21 & 0.14 & 0.11 & 0.12 & 0.40 \\
\hline $\mathrm{Na}$ & 0.71 & 1.01 & 0.85 & 0.75 & 0.72 \\
\hline K & 0.05 & 0.06 & 0.06 & 0.05 & 0.07 \\
\hline $\mathrm{Mg}$ & 0.35 & 0.34 & 0.32 & 0.33 & 0.32 \\
\hline $\mathrm{S}$ & 0.06 & 0.05 & 0.05 & 0.04 & 0.05 \\
\hline $\mathrm{Cl}$ & 0.64 & 0.73 & 0.68 & 0.71 & 0.69 \\
\hline $\mathrm{Fe}$ & 0.12 & 0.08 & 0.04 & 0.05 & 0.04 \\
\hline $\mathrm{Al}$ & 0.11 & 0.09 & 0.07 & 0.06 & 0.18 \\
\hline $\mathrm{Zn}$ & 0.073 & 0.271 & 0.132 & 0.098 & 0.09 \\
\hline $\mathrm{Sr}$ & 0.056 & 0.050 & 0.064 & 0.077 & 0.077 \\
\hline $\mathrm{Zr}$ & 0.033 & 0.012 & 0.031 & 0.00 & 0.00 \\
\hline $\mathrm{Cu}$ & 0.00 & 0.020 & 0.03 & 0.012 & 0.00 \\
\hline $\mathrm{La}$ & 0.00 & 0.00 & 0.00 & 0.00 & 0.105 \\
\hline $\mathbf{F}$ & 0.00 & 0.25 & 0.16 & 0.08 & 0.05 \\
\hline LOI* & 15.12 & 14.12 & 12.56 & 15.36 & 14.27 \\
\hline
\end{tabular}

* LOI refers to the test named "Loss on Ignition" that is carried out by strongly heating (igniting) a sample of the material at a specified temperature to allow volatile substances to escape until its mass ceases to change.

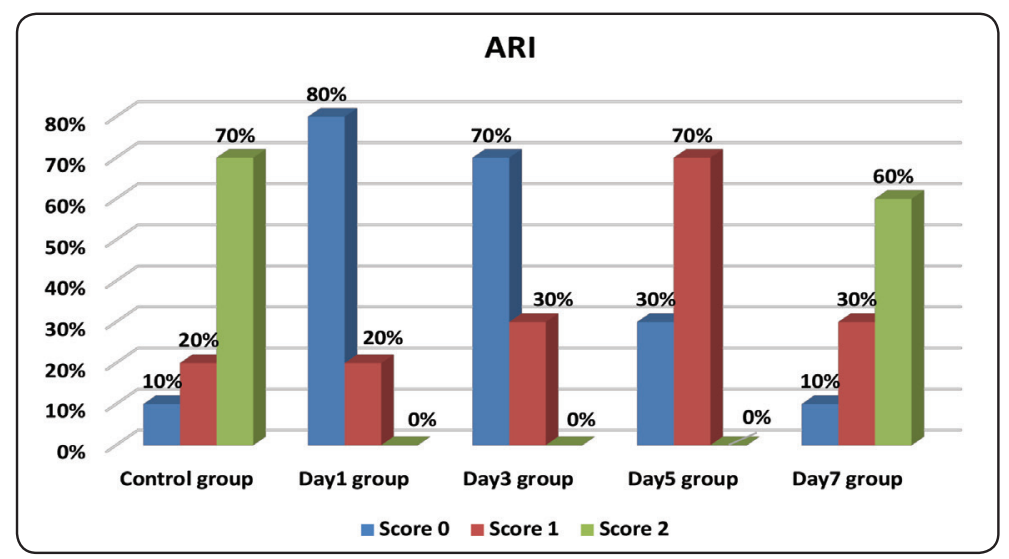

Fig. (3) Adhesive remnant index (ARI) scores of control and fluoridated groups. 

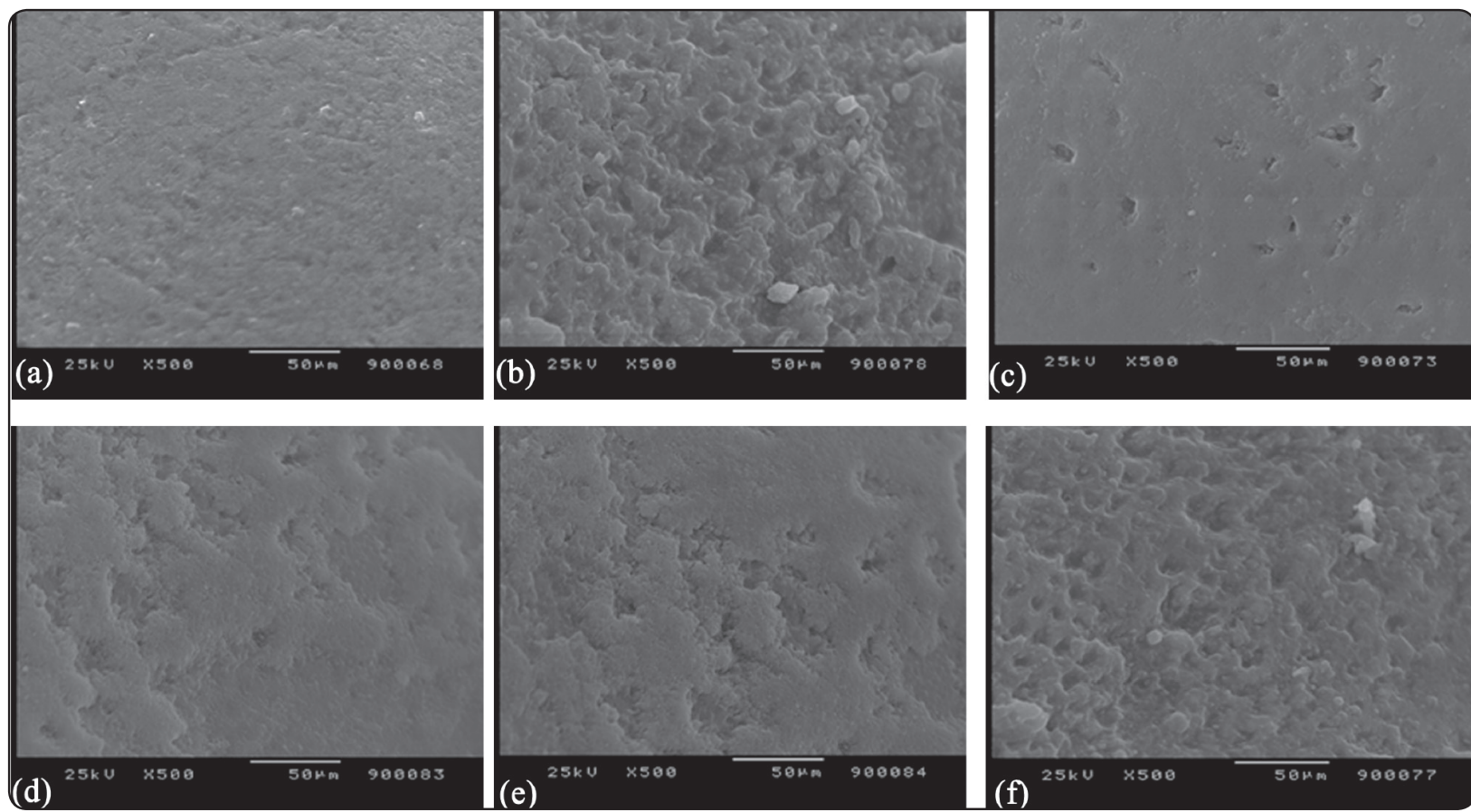

Fig. (4) Scanning electron micrographs (500 x) showing: a) sound untreated (no fluoridation, no etching) smooth enamel surface with small pits, b) enamel surface of control group (etched without fluoridation) demonstrating a honeycomb appearance, $\mathrm{c}$ ) enamel surface of Day1 group (fluoridated and etched) showing an intact fluoride layer covering the entire enamel surface with a few irregular pits, d) enamel surface of Day3 group (fluoridated and etched) showing a fluoride layer covering the entire enamel surface with more and larger pits after some dissolution of the fluoride layer, e) enamel surface of Day5 group (fluoridated and etched) showing more dissolution of the fluoride layer with some exposure of the enamel surface and f) enamel surface of Day7 group (fluoridated and etched) showing a nearly complete dissolution of the fluoride layer and the enamel surface showing the honeycomb appearance like that seen in the control group.



Fig. (5) Linear regression analysis showed strong negative correlation $\left(\mathrm{R}^{2}=0.959\right)$ between shear bond strength (MPa) of fluoridated groups and fluoride content (wt\%) as stated by the XRF analysis.

\section{DISCUSSION}

Failure of bracket/tooth bonding is an annoying occurrence in orthodontic practice. As the location of bond failure may indicate the probable cause, it is very essential to study the significance of "bond strength" in a clinical application. Bonding in orthodontics can be investigated by one of three ways ${ }^{[25]}$ : i) in vivo, using controlled clinical models, ii) in vitro, using simulated clinical models, and iii) isolated substrate models, in which bonding of an adhesive to a bracket or tooth structure is studied independently. In the current study, we studied the bond strength, in vitro, on clinically relevant models.

As there has not been definite specification/ protocol (such as ISO, ANSI, ADA ASTM) for orthodontic bond strength testing to follow ${ }^{[25,26]}$, 
we did our best to get the most reliable results in this study. In terms of bonding to the orthodontic adhesives, it has been reported that ceramic brackets adhere more favorably than the metallic brackets $\mathrm{do}^{[27,28]}$. Authors attributed this superior adhesion to many factors: i) ceramic brackets allow for more light to pass for polymerizing the underlying resin adhesive giving stronger bond ${ }^{[29]}$, ii) availability of various base designs that increase the mechanical interlocking such as beads, grooves or pits ${ }^{[30]}$, in addition to other designs with a chemical coating, such as silane coupling agent, to enhance chemical bonding between the adhesive and the bracket base ${ }^{[31]}$ and iii) the composition nature of ceramic brackets that are of two types; monocrystalline and polycrystalline aluminum oxide $\left(\mathrm{Al}_{2} \mathrm{O}_{3}\right)$, with better performance recorded for monocrystalline brackets because of better heat treatment that minimizes stress-inducing impurities and imperfections that can serve as foci for crack propagation under stress and compromise the bracket during clinical use ${ }^{[32}$, ${ }^{33]}$. So, in the present study, monocrystalline ceramic brackets (Perfect Clear, Orthodontic Sapphire Ceramic Brackets, HUBIT Co., Ltd. South Korea) with beads base design were used. Using ceramic brackets in the present study was quietly helpful to minimize failure at the bracket/adhesive interface and maximize failure at tooth/adhesive interface at the time of specimens debonding.

As the main purpose of this study was to find out the relationship between the SBS and fluoride content either adsorbed onto the tooth surface or absorbed into the tooth, two means of investigation were used; SEM and XRF analysis. The SEM is a well-established and very effective method to provide information about the surface features and texture, shape, size and arrangement of the particles lying on the sample's surface ${ }^{[34]}$. The kind of elements and their percentage in the tooth structure were determined by the XRF analysis. The range of storage time chosen in the current study was based on literature studies. The effect of topical fluoride for $24 \mathrm{~h}$ on bracket bond strength was investigated by many researchers. Some studies reported no effect of topical fluoride after seven days storage; therefore, our study groups were stored for a time range starting with $24 \mathrm{~h}$ and ending with seven days ${ }^{[7]}$.

As there were statistically significant differences between SBS means of studied groups, the first null hypothesis was rejected. Fluoridated groups (Day1, Day3 and Day5) showed significantly lower SBS than the control group. Day7 group, however, recorded comparable SBS to that of the control one $(\mathrm{p}=0.773)$. This might be attributed to the effect of calcium fluoride layer $\left(\mathrm{CaF}_{2}\right)$ that is formed on top of the enamel surface that reduced penetration of adhesive resin into enamel surface. In addition, deposition of fluoride ions into hydroxyapatite crystals could form fluoroapatite crystals that are wellknown with their greater resistance to acid-etching ${ }^{[35]}$. In agreement with our findings, Takahashi et al. ${ }^{[36]}$ found that when $0.2 \%$ sodium fluoride $(0.09 \%$ fluoride) was added to $30 \%$ phosphoric acid, there was a significant reduction in the bond strength. Also, Leodido et al. ${ }^{[37]}$ conducted an in vitro study to evaluate the effect of different fluoride solutions - acidulated phosphate fluoride, neutral fluoride and sodium fluoride varnish - on SBS of orthodontic brackets that were attached directly after fluoride treatment. Authors noticed that all studied types of fluoride solutions promoted a decrease in SBS. Moreover, when a fluoride varnish (Clinpro ${ }^{\mathrm{TM}}$ White varnish, 2.23\% F, 3M, UniketLandsberg, Germany) or a fluoride gel (Elmex Fluid, GABA Basel, Switzerland) was applied to the enamel surface, a reduction in the bond strength was evident ${ }^{[13]}$.

Within fluoridated groups, a systematic enhancement of SBS was observed with longer storage time in artificial saliva (Figure 2). Longer storage time allowed for the $\mathrm{CaF}_{2}$ layer to be washed away and more enamel surface became exposed for bonding giving greater SBS. This could simply explain the increased SBS of Day5 and Day7 groups compared to that of Day1 and Day3 groups. The recovery of SBS after storage in artificial saliva for 7 days to be comparable to that of the unfluoridated group 
is consistent with the result reported by Ortiz-Ruiz et al. ${ }^{[7]}$.

According to the ARI system applied in this study, majority of specimens of control group exhibited score $2(70 \%)$ with more than $50 \%$ of the adhesive remained on the tooth surface. The Day7 group was the only fluoridated group that demonstrated ARI scores close to that of the control group, where six specimens (60\%) were given score 2 . For Day1 and Day3 groups, majority of specimens showed no adhesive remaining on the tooth surface (score 0 ). This was the case in eight specimens of Day1 group and seven specimens of Day3 group. The remaining two specimens of Day1 and three specimens of Day3 were given score 1. For Day5 group, seven specimens (70\%) were given score 1 and three $(30 \%)$ were given score 0 . None of specimens of all groups, however, showed ARI at score 3 indicating good adhesion between the ceramic brackets and the orthodontic adhesive used in this study.

The results of SBS testing and ARI findings were almost reflected in the SEM investigation (Figure 4) where the fluoride layer painted on the enamel surface acted as a barrier hindering the effectiveness of etching acid and minimizing the number of micropores into which the resin can penetrate to enhance bonding. This was most prominent in Day1 group. As storage in artificial saliva caused the fluoride to dissolve, Day3 and Day5 groups showed discontinuous fluoride layer and more roughness on the enamel surface. Day7 group presented an SEM image almost similar to that of control group because of nearly complete disappearance of the fluoride layer.

From the results of both SBS and XRF analysis, it is clear that there is, for the fluoridated groups, a negative correlation between the two variables; therefore, the second null hypothesis was rejected as well. Variation in the fluoride content of fluoridated groups could be attributed to different storage time. When the fluoridated samples were left in artificial saliva for a longer time (samples of Day7 group) more fluoride ions were dissolved from the tooth surface than those left for a shorter time (samples of Day1 and Day3 groups). More removal of fluoride ions from the tooth surface resulted in better etching and greater SBS. According to these findings, both the SEM investigation and XRF analysis confirmed the negative effect of fluoride application on bond strength of orthodontic brackets.

Despite the reduction of SBS with fluoride application, the lowest value recorded in our study, for Day1 group (6.61 MPa), was still within the range of the bond strength (5.9 to $7.8 \mathrm{MPa}$ ) clinically acceptable to retain the bracket to accomplish the orthodontic treatment ${ }^{[38,39]}$. From a clinical point of view, it can be recommended that if fluoride application is mandatory for some orthodontic patients to prevent initiation or stop propagation of dental caries, bonding of orthodontic brackets should be delayed for some days after fluoride application. In this way, fluoride ions will be minimal on the enamel surface because of solubility in oral fluids, thus adequate etching of the tooth surface will be possible giving enough bonding to maintain the brackets on the tooth surface for the treatment time. While still having some fluoride ions, though minimal, specimens of Day7 group demonstrated SBS comparable to that of unfluoridated group. This means that bonding orthodontic brackets to fluoridated teeth seven days after fluoride treatment can give us two advantages; anticariogenic activity because of the fluoride ions contained in the tooth surface and adequate bonding characteristics.

\section{CONCLUSIONS}

- As confirmed by SEM and XRF analysis, fluoride reduced SBS of orthodontic brackets in the first few days of fluoride treatment.

- As fluoride dissolved in artificial saliva after a period of time, SBS recovered to normal values.

- Bonding orthodontic brackets to fluoridated teeth 7 days after treatment can provide adequate bonding besides the anticariogenic activity. 


\section{REFERENCES}

1. Stangel I, Ellis TH, Sacher E. Adhesion to Tooth Structure Mediated by Contemporary Bonding Systems. Dental Clinics of North America, 2007; 51:677-694.

2. Armstrong S, Geraldeli S, Maia R, Raposo LHA, Soares CJ, Yamagawa J. Adhesion to tooth structure: A critical review of "micro" bond strength test methods. Dental Materials 2010; 26:50-62.

3. Carvalho RM, Manso AP, Geraldeli S, Tay FR, Pashley DH. Durability of bonds and clinical success of adhesive restorations. Dental Materials, 2012; 28:72-86.

4. Birdsall J, Hunt NP, Sabbah W, Moseley HC. Accuracy of positioning three types of self-ligating brackets compared with a conventionally ligating bracket. J Ortho, 2012; 30:34-42.

5. Valletta R, Prisco D, Santis R, Ambrósio L, Martina R. Evaluation of the debonding strength of orthodontic brackets using three different bonding systems. Eur J Orthod. 2007; 29:571-7.

6. Kimura T, Dunn WJ, Taloumis LJ. Effect of fluoride varnish on the in vitro bond strength of orthodontic brackets using a self-etching primer system. American Journal of Orthodontics and Dentofacial Orthopedics, 2004; 125:351-356.

7. Ortiz-Ruiz AJ, Munoz-Gomez IJ, Perez-Pardo A, GermanCecilia C, Martinez-Beneyto Y, Vicente A. Influence of fluoride varnish on shear bond strength of a universal adhesive on intact and demineralized enamel. Odontology 2018; 106:460-468.

8. Ten Cate JM, Duijsters PPE. Influence of fluoride in solution on tooth demineralization. I. Chemical data. Caries Res.1983; 17:193-199.

9. Christoffersen J, Christoffersen MR, Kibalczyc W, Perdok WG. Kinetics of dissolution and growth of calcium fluoride and effects of phosphate. Acta Odontologica Scandinavica, 1988; 46:325-36.

10. Chersoni S, Bertacci A, Pashley DH, Tay FR, Montebugnoli L, Prati C. In vivo effects of fluoride on enamel permeability. Clin Oral Investig. 2011; 15:443-449

11. Schmidlin PR, Zehnder M, Pasqualetti T, Imfeld T, Besek MJ. Penetration of a bonding agent into de- and remineralized enamel in vitro. J Adhes Dent. 2004; 6:111-115.

12. Xiaojun D, Jing L, Xuehua G, Hong R, Youcheng Y, Zhangyu G, et al. Effects of CPP-ACP paste on the shear bond strength of orthodontic brackets. Angle Orthod. 2009; 79:945-950.
13. Attin R, Stawarczyk B, Keçik D, Knosel M, Wiechmann $\mathrm{D}$, Attin T. Shear bond strength of brackets to demineralize enamel after different pretreatment methods. Angle Orthod. 2012; 82:56-61.

14. Choi S, Cheong Y, Lee G-J, Park H-K. Effect of fluoride pretreatment on primary and permanent tooth surfaces by acid-etching. Scanning. 2010; 32:375-382.

15. Baysal A, Uysal T. Do enamel microabrasion and casein phosphopeptide-amorphous calcium phosphate affect shear bond strength of orthodontic brackets bonded to a demineralized enamel surface? Angle Orthod, 2012; $82: 36-41$

16. Akin M, Baka ZM, Ileri Z, Basciftci FA. Can demineralized enamel surfaces be bonded safely? Acta Odontol Scand. 2014; 72:283-289.

17. Uo M, Wada T, Sugiyama T. Applications of X-ray fluorescence analysis (XRF) to dental and medical specimens. Japanese Dental Science Review, 2015; 51:2-9.

18. Brouwer P. Theory of XRF. 3rd edition 2010 by PANalytical, Netherlands; p: 8-11.

19. Baranowska I, Barchanski L, Bak M, Smolec B, Mzyk Z. $\mathrm{X}$-ray fluorescence spectrometry in multielemental analysis of hair and teeth. Pol J Environ Stud 2004; 13:639-646.

20. International Organization for Standardization. ISO TS 11405. Dental materials-guidance on testing of adhesion to tooth structure. Geneva: Switzerland; 2015.

21. Geramipanah F, Majidpour M, Sadighpour L, Fard M. Effect of artificial saliva and $\mathrm{pH}$ on shear bond strength of resin cements to zirconia-based ceramic. Eur J Prosthodont Rest Dent, 2013; 21: 1-4.

22. Artun J, Bergland S. Clinical trials with crystal growth conditioning as an alternative to acid etch enamel pretreatment. Am J Orthod, 1984; 85:333-340.

23. Lalani N, Foley TF, Voth R, Banting D, Mamandras AH. Polymerization with the argon laser: curing time and shear bond strength. Angle Orthod, 1999; 69:525-534.

24. American Society for Testing and Materials. ASTM E1621, 2013 - Elemental Analysis by Wavelength Dispersive X-Ray Fluorescence Spectrometry.

25. Powers JM, Kim HB, Turner DS. Orthodontic adhesives and bond strength testing. Seminars in Orthodontics, 1997; $3: 147-156$.

26. Katona TR, Long RW. Effect of loading mode on bond strength of orthodontic brackets bonded with 2 systems. American Journal of Orthodontics and Dentofacial Orthopedics, 2006; 129:60-64. 
27. Ansari MY, Agarwal DK, Gupta A, Bhattacharya P, Ansar J, Bhandari R. Shear bond strength of ceramic brackets with different base designs: Comparative in-vitro study. Journal of clinical and diagnostic research: JCDR. 2016; 10:ZC64.

28. Gwinnett AJ. A comparison of shear bond strengths of metal and ceramic brackets. Am J Orthod Dentofacial Orthop, 1988; 93:346-348.

29. Joseph VP, Rossouw E. The shear bond strengths of stainless steel and ceramic brackets used with chemically and light-activated composite resins. Am J Orthod Dentofacial Orthop. 1990; 97:121-125.

30. Gautam P, Valiathan A. Ceramic brackets: in search of an ideal! Trends in Biomaterials and Artificial Organs, 2007; 20:122-126.

31. Kukiattrakoon B, Samruajbenjakul B. Shear bond strength of ceramic brackets with various base designs bonded to aluminous and fluorapatite ceramics. European Journal of Orthodontics, 2010; 32:87-93.

32. Ødegaard J, Segner D. The use of visible light curing composites in bonding ceramic brackets. Am J Orthod Dentofacial Orthop, 1990; 97:183-193.
33. Viazis AD, Cavanaugh G, Bevis RR. Bond strength of ceramic brackets under shear stress: an in vitro report. Am J Orthod Dentofac Orthop, 1990; 98: 214-221.

34. Hayes TL, Pease RFW. The scanning electron microscope: Principles and applications in biology and medicine. Advances in biological and medical physics, 1998; 12:85-117.

35. Ganss C, Klimek J, Schaffer U, Spall T. Effectiveness of two fluoridation measures on erosion progression in human enamel and dentine in vitro. Caries Res 2001; 35:325-330.

36. Takahashi Y, Arakawa Y, Matsukubo T, Takeuchi M. The effect of sodium fluoride in acid etching solution on sealant bond and fluoride uptake. J Dent Res, 1980; 59:625-630.

37. Leodido GR, Fernandes HO, Tonetto MR, Presosto CD, Bandeca MC, Firoozmand LM. Effect of Fluoride Solutions on the Shear Bond Strength of Orthodontic Brackets. Braz Dent J, 2012; 23: 698-702.

38. Tecco S, Traini T, Caputi S, Festa F, de Luca V, D'Attilio M. A New One-Step Dental Flowable Composite for Orthodontic Use: An In Vitro Bond Strength Study. Angle Orthod, 2005; 75:574-579.

39. Albaladejo A, Montero J, de Diego RG, López-Valverde A. Effect of adhesive application prior to bracket bonding with flowable composites. Angle Orthod, 2011; 81:716-720. 